\title{
FINITE ELEMENT ANALYSIS OF STENT EXPANSION: INFLUENCE OF STENT GEOMETRY ON PERFORMANCE PARAMETERS
}

\author{
I.V. Gomes ${ }^{1}$, H. Puga ${ }^{1}$, J.L. Alves ${ }^{1}$, J.C.P. Claro ${ }^{1}$, \\ ${ }^{1}$ CMEMS - Center for Microelectromechanical Systems \\ Department of Mechanical Engineering, University of Minho, Campus of Azurém, 4800-058 \\ Guimarães, Portugal
}

\begin{abstract}
Ischaemic heart diseases are a global health problem that have been increasing in the recent years [1]. Most of ischaemic heart diseases is caused by atherosclerosis, a clinical pathology characterized by the deposition of fatty material on the arteries walls what may lead to thickening, hardening and blocking of these blood vessels, reducing the blood flow. This situation can cause heart attack, stroke and, in extreme cases, death $[1,2]$.
\end{abstract}

Procedures such as balloon angioplasty and stenting, bypass surgery and atherectomy may be used to repair a blocked artery. Balloon expandable stents are the most used technique [1].

Materials such as stainless steel, Niobium and CoCr alloys are the most used in stents manufacturing. However, in the last years, studies have been directed to the search and development of new materials, mainly biodegradables, once these solutions fulfil some unmet medical needs [3]. The application of biodegradable stents dispenses a second procedure to remove the device, once this is absorbed over time. Magnesium alloys are one of the options.

The main purpose of this work is the study of an AZ31 magnesium alloy stent expansion behaviour, without considering the contact definitions, by using the finite element method.

A simple geometry composed by lozenges was modelled and simulated in order to study the required expansion pressure, equivalent plastic strain and other performance parameters such as dogboning, foreshortening and recoil. The model has an inner diameter of $0,74 \mathrm{~mm}$ and a length of $10 \mathrm{~mm}$ and its results were compared with those of a Palmaz-Schatz model, aiming to conclude about which geometry presents better performance. Both models were tested for thicknesses of $0.1 \mathrm{~mm}$ and $0.2 \mathrm{~mm}$.

The obtained results showed that Lozenge model requires higher expansion pressures than Palmaz-Schatz model and has worse performance in terms of foreshortening. In terms of dogboning, the Lozenge model presents better results, especially for $0.1 \mathrm{~mm}$ of thickness. In what concerns to recoil phenomenon, considering the longitudinal recoil, Palmaz-Schatz model presents most favourable values, although, for central and distal recoil, Lozenge model has better results.

\section{INTRODUCTION}

In the last years, the number of cases of ischemic heart diseases have been increasing, being one of the main causes of death in the world. Most of these problems are caused by atherosclerosis, a pathology characterized by the deposition of fatty material along the arteries walls, what may lead to thicken, harden or even block of the blood vessels, resulting in an insufficient blood flow to the heart. Problems such as heart attack, stroke and even death may occur as consequence of the reduction of the blood flow [4,5].
Balloon angioplasty with stenting, bypass surgery and atherectomy are some procedures that can be used to revascularise a blocked artery [5]. The implantation of a stent to reopen a blood vessel and restore the normal blood flow is one of the most popular techniques [6].

Typically, the stent is crimped on a deflated balloon and placed into the artery by a catheter system. After this stage, the balloon is inflated, causing the gradual stent deformation until the target deployment diameter be reached. The balloon returns to its original shape and is extracted out with the catheter system [7].

Nowadays, the stent technology is based on the implantation of permanent devices which are made from corrosion-resistant metals and can cause problems as long-term endothelial dysfunction, permanent physical irritation and chronic inflammatory local reactions, among others [8]. This solution has some drawbacks in its widespread application because of its long-term biological incompatibility [9].

The stent scaffolding effect must last for 6-12 months in order to allow the artery remodeling and healing. When this process is concluded, the implant is not needed anymore, so biodegradable materials are an interesting solution: after the accomplishment of their purpose, they degrade themselves without risks for the human organism [10]. Thus, the materials used in biodegradable stents manufacturing must be biocompatible and capable of staying in the human organism while the scaffolding effect is needed. As the stent will be degraded by its interaction with the human organism, the products of this process must also be biocompatible [11].

Magnesium alloys are one of the most popular options for biodegradable stents. The biocompatibility of these materials is well-known [10] and the magnesium is an essential element of the human organism. Besides this, magnesium does not represent any carcinogenic risk [12]. In order to improve the mechanical properties, magnesium alloys are the preferred option

\section{Simulation Strategies}

The use of Finite Element Analysis provides information about the biomechanical behavior of the system during the stent implantation process. This technique is faster and less expensive and invasive than experimental methods [5].

Several simulation strategies with different complexity and accuracy levels were proposed by different authors. In the first approaches, the process was simulated using only the stent model, without considering the contact effects with the vessels and the plaque [14]. In order to achieve better results, more complex models were tested, including different 
elements of the real system (blood vessel, plaque and balloon).

Gervaso et al.[15] studied both influence of the simulated system and load application conditions in order to conclude about which method allowed to reach results consonant with those proposed by the manufacturers. Three different methods were tested: i) pressure application on the stent inner surface with no balloon; ii) free rubber cylinder inside the stent and iii) folded balloon. The obtained results showed that only the model where the balloon expansion inside the stent promoted its expansion provided similar to manufacturers data results. These results were in agreement with those obtained by M. de Beule et al. [16].

In what concerns to the material models of the system constituents, some formulations were proposed and studied, namely linear isotropic [18] and hyperplastic material models [3,22], using Mooney-Rivlin description. The correct definition of material properties such as Young's modulus, density and Poisson's ration is crucial to reach reliable results. Once this is a large deformation problem, it is also important the correct definition of the hardening model.

Besides the simulated system and the material model formulation, the definition of boundary conditions is an important step of the pre-processing stage, being necessary to accurately define the constraints and loading conditions, which influences the results applicability to the reality and its reliability.

Once again, many studies were developed in this scope.

Ning Li and Yuanxian Gu [21] only considered the large deformation of the stent, neglecting all the contacts and its effects on the expansion behavior of the device.

A more complex study was carried on by Misagh Imani et al [1], where the contact between the different elements of the system were considered, being assumed that both ends of the balloon were completely fixed and only the nodes of the artery ends were able to move radially. The plaque and the artery were defined as bounded. The load application was defined by steps: i) first, it was applied a constant pressure equal to $13.3 \mathrm{kPa}$ in both artery and plaque, in order to simulate a 100 $\mathrm{mmHg}$ blood pressure [19]; ii) in a second stage, it was applied a constant pressure in the balloon inner surface, leading to its expansion.

Another study was developed by Schiavone and Zhao [22] that defined the constituents of the system (stent, artery and plaque) as a hard contact, characterized by a friction coefficient of 0.25 . These authors also studied different combinations of boundary conditions, mainly constraints, in order to study its influence on stent expansion. The balloon was fixed in its ends to simulate its connection to the catheter used in the stenting process, the axial moves were blocked and radial expansion was allowed.

The geometry optimization is one of the most important aims of stenting process studies, however, there are few works in this scope, most of all developed in clinical context.

Aiming to study and compare different solutions, Misagh Imani et al. [1] simulated different stent geometries in order to analyze its behavior and compare the obtained results in terms of stress distribution, radial expansion and dogboning.
Shankaran and Karrupaswamy [2] developed a more efficient optimization method by the parametrization of some variables such as thickness, whose values varied in a defined range, allowing to conclude about which solution is more advantageous in terms of the referred parameters.

\section{SIMULATION MODEL}

The good performance of the stent is assured by its capability of suffering a plastic deformation that allows the reopen of a blocked vessel, reason why this is a large deformation problem, implying a non-linear analysis.

This work presents the simulation of radial expansion of two different stent geometries, Lozenge and Palmaz-Schatz, in order to evaluate the required expansion pressure, the equivalent plastic strain imposed by the large deformation and the dogboning, foreshortening and recoil phenomena.

The stent radial expansion is a structural mechanics problem and it was studied using a quasi-static analysis in COMSOL Multiphysics.

\section{A. Geometric Models and Material Properties}

Palmaz-Schatz model is characterized by its straight lines, which form a grid that allows the uniform load application in the stent inner surface.

Lozenge model is a simple geometry which resembles a lozenge mesh.

Both models (Fig. 1) have inner radius of $0.74 \mathrm{~mm}$, length of $10 \mathrm{~mm}$ and thickness of 0.1 and $0.2 \mathrm{~mm}$. (a)

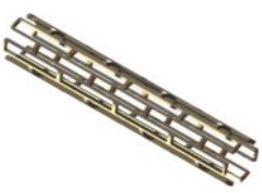

Figure 1. Stent geometries (a) Palmaz-Schtaz; (b) Lozenge
The selected material is a AZ31 magnesium alloy, defined as homogeneous, isotropic and elastoplastic, being used the plasticity model of Von Mises for large deformations. It is used an isotropic hardening model based on stress-strain material curve data. The curve that describes the plastic behavior of the material is defined by (1).

$$
Y=Y_{0}+\left(Y_{\text {sat }}-Y_{0}\right)\left(1-\exp \left(-C_{y} \times \varepsilon_{0}\right)\right.
$$

Where $Y_{0}$ is the yield stress, equal to $174.8 \mathrm{MPa}, Y_{\text {sat }}$ is the plasticity stress, equal to $315.6 \mathrm{MPa}, C_{y}$ is a constant, equal to 16.3 and $\varepsilon_{0}$ is the plastic strain.

\section{B. Boundary Conditions and Loading Application}

Due the radial and longitudinal symmetries, only a one twelfth of the Lozenge model and a one twenty-fourth of the Palmaz-Schatz model are simulated.

The symmetry conditions are applied at one end of the models and at its lateral surfaces, which allows its radial expansion and longitudinal deformation as result of it.

The process simulation has two different stages: i) balloon insufflation inside the stent until the target diameter is 
reached; ii) reduction of the applied pressure to zero, which allows the recoil phenomenon of both artery and stent.

In this work, it is studied the referred parameters for a radial expansion equal to $2 \mathrm{~mm}$ which corresponds to $170 \%$ of expansion relatively to the initial radius.

\section{RESUlTS}

\section{A. Radial Expansion and Required Pressure}

The geometries behavior was studied considering a radial expansion equal to $2 \mathrm{~mm}$ which means that its final diameter is $4 \mathrm{~mm}$. The obtained results of required pressure to promote the referred deformation are presented in Fig. 2.

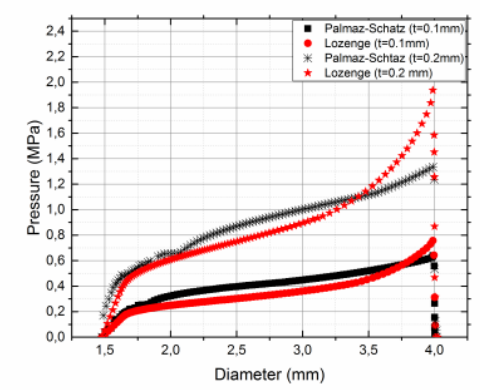

Figure 2. Relation between expanded diameter and required pressure for thickness od $0.1 \mathrm{~mm}$ and $0.2 \mathrm{~mm}$

By the analysis of the obtained results, it is possible to conclude that, considering the same thickness, the Lozenge model requires higher expansion pressure than the PalmazSchatz model to promote the desired radial expansion.

The increase in thickness leads to the increase of the required expansion pressure in both models.

Considering a thickness of $0.1 \mathrm{~mm}$ and an expanded diameter equals to $4 \mathrm{~mm}$, the Lozenge model requires 0.76 $\mathrm{MPa}$ and the Palmaz-Schatz requires $0.62 \mathrm{MPa}$, which represents a difference higher than $22.5 \%$.

\section{B. Dogboning}

Dogboning is the used term of art to refer to the expansion of a stent at the proximal and distal ends, assuming a shape near to that of a dog bone.

This parameter is used to evaluate the stent performance, once this shape can compromise the vessel integrity and, eventually, cause injuries.

In terms of dogboning, the obtained results are presented in Fig. 3.

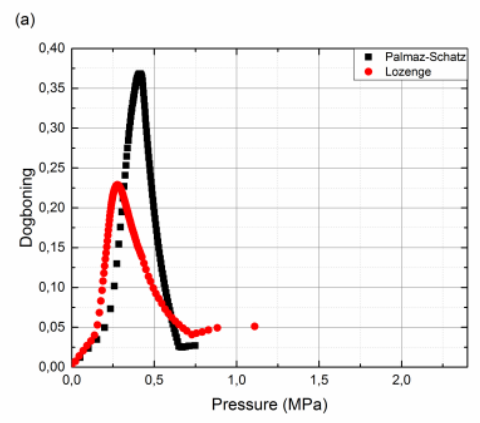

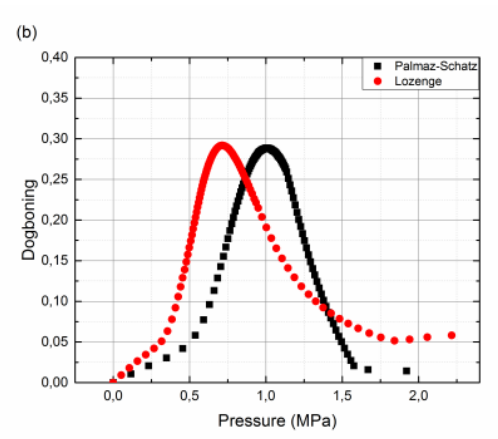

Figure 3. Relation between dogboning and required expansion pressure for thickness of (a) $0.1 \mathrm{~mm}$; (b) $0.2 \mathrm{~mm}$

The obtained results analysis suggests that, for a thickness equals to $0.1 \mathrm{~mm}$, the Palmaz-Schatz model presents higher values of dogboning than the Lozenge model, which means that the first model is more propitious to provoke injuries in the blood vessels than the second model. For a thickness of $0.2 \mathrm{~mm}$, the dogboning values are similar for both models, being higher than those of Lozenge model and thickness of $0.1 \mathrm{~mm}$ and lower than those of Palmaz-Schatz model and thickness of $0.1 \mathrm{~mm}$.

\section{Foreshortening}

Foreshortening is a performance parameter that corresponds to the percentage by which the length of the stent decreases from its initial state (initial length).

The Fig. 4 presents the obtained results in terms of foreshortening for both models and thicknesses.
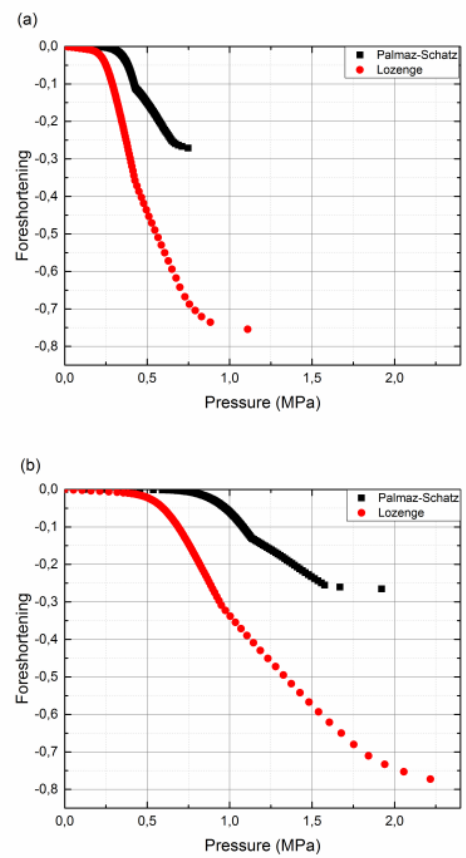

Figure 4. Relation between foreshortening and required expansion pressure for thickness of (a) $0.1 \mathrm{~mm}$; (b) $0.2 \mathrm{~mm}$

By the analysis of the data provided by Fig. 4, it is possible to conclude that Lozenge model presents higher values of foreshortening than Palmaz-Schatz model, which means that it suffers a higher reduction of length as its diameter expands. 
The maximum value of foreshortening is similar for both values of thickness.

Considering a thickness of $0.1 \mathrm{~mm}$, the reduction of the stent length is approximately $75 \%$ for Lozenge model and $25 \%$ for Palmaz-Schatz model.

\section{Recoil}

The percentage by which the diameter of a stent decreases from its expanded diameter (when the balloon is inflated at the nominal pressure) to its relaxed diameter (when the balloon is retrieved from the stent) is designed recoil.

The obtained results in terms of longitudinal, central and distal recoil are presented in Table I.

TABLE I. RECOIL EVALUATION FOR PALMAZ-SCHATZ AND LOZENGE MODELS FOR BOTH THICKNESSES

\begin{tabular}{ccccc}
\hline \multirow{2}{*}{ Recoil Evaluation } & \multicolumn{2}{c}{ Palmaz-Schatz Model } & \multicolumn{2}{c}{ Lozenge Model } \\
\cline { 2 - 5 } & $\mathbf{0 . 1 m m}$ & $\mathbf{0 . 2 m m}$ & $\mathbf{0 . 1 m m}$ & $\mathbf{0 . 2 m m}$ \\
\hline Longitudinal Recoil & $-4.21 \%$ & $-3.8 \%$ & $-11.8 \%$ & $-11.5 \%$ \\
\hline Distal Recoil & $4.03 \%$ & $3.83 \%$ & $3.27 \%$ & $2.7 \%$ \\
\hline Central Recoil & $4.19 \%$ & $4.1 \%$ & $4.12 \%$ & $3.5 \%$ \\
\hline
\end{tabular}

In terms of recoil evaluation, the obtained results show that the longitudinal recoil is higher for Lozenge model than for Palmaz-Schatz model, even though distal and central recoil values are lower for Lozenge model. Besides this, for higher values of thickness, the recoil values are lower for both models.

\section{CONCLUSIONS}

In this study, the required expansion pressure to promote the stent radial deformation from radius of $0,74 \mathrm{~mm}$ to $2 \mathrm{~mm}$ was studied for Palmaz-Schatz and Lozenge models, as well as its performance in terms of dogboning, foreshortening and recoil.

By the obtained results analysis, it is possible to conclude:

- Greater thicknesses require higher expansion pressures;

- Palmaz-Schatz model requires higher expansion pressures than Lozenge model to promote the same radial expansion $(4 \mathrm{~mm})$;

- In terms of dogboning, considering a thickness of $0.1 \mathrm{~mm}$, Lozenge model presents better performance than Palmaz-Schatz model. For thickness equal to $0.2 \mathrm{~mm}$, the obtained results are similar for both models;

- For both values of thickness, Palmaz-Schatz presents better performance than Lozenge in terms of foreshortening;

- In what concerns to stent recoil, Palmaz-Schatz presents better results for longitudinal recoil but poorer ones for distal and central recoil.

\section{REFERENCES}

[1] M. Imani, A. M. Goudarzi, D. D. Ganji, and A. L. Aghili, "The comprehensive finite element model for stenting: The influence of stent design on the outcome after coronary stent placement," J. Theor. Appl. Mech., vol. 51, no. 3, pp. 639-648, 2013.
[2] K. Shankaran and S. Karrupaswamy, "Parameterization and Optimization of balloon expandable Stent," in Proceedings of the SIMULIA Community Conference, 2012.

[3] I. Pericevic, C. Lally, D. Toner, and D. J. Kelly, "The influence of plaque composition on underlying arterial wall stress during stent expansion: The case for lesion-specific stents," Med. Eng. Phys., vol. 31, no. 4, pp. 428-433, May 2009.

[4] K. Shankaran and S. Karrupaswamy, "Parameterization and Optimization of balloon expandable Stent," in Proceedings of the SIMULIA Community Conference, 2012.

[5] M. Imani, A. M. Goudarzi, D. D. Ganji, and A. L. Aghili, "The comprehensive finite element model for stenting: The influence of stent design on the outcome after coronary stent placement," J. Theor. Appl. Mech., vol. 51, no. 3, pp. 639-648, 2013.

[6] A. Schuessler, U. Bayer, G. Siekmeyer, R. Steegmueller, M. Strobel, and A. Schuessler, "Manufacturing of stents: optimize the stent with new manufacturing technologies," in 5th European symposium of vascular biomaterials ESVB, 2007.

[7] N. Li and Y. Gu, "Parametric design analysis and shape optimization of coronary arteries stent structure," Proc. 6th World Congr. Struct. Multidiscip. Optim. Rio Jan., vol. 30, 2005.

[8] P. Erne, M. Schier, and T. J. Resink, "The Road to Bioabsorbable Stents: Reaching Clinical Reality?," Cardiovasc. Intervent. Radiol., vol. 29, no. 1, pp. 11-16, Feb. 2006.

[9] J. Li, F. Zheng, X. Qiu, P. Wan, L. Tan, and K. Yang, "Finite element analyses for optimization design of biodegradable magnesium alloy stent," Mater. Sci. Eng. C, vol. 42, pp. 705-714, Sep. 2014.

[10] M. Moravej and D. Mantovani, "Biodegradable Metals for Cardiovascular Stent Application: Interests and New Opportunities," Int. J. Mol. Sci., vol. 12, no. 12, pp. 4250-4270, Jun. 2011.

[11] S. Saito, "New horizon of bioabdsorbable stent," Catheter Cardiovasc. Interv., vol. 66, pp. 595-596, 2005.

[12] M. Morajev, S. Amira, F. Prima, A. Rahem, M. Fiset, and D. Montovani, "Effect of electrodeposition current density on the microstructure and the degradation of electroformed iron for degradable stents."

[13] L. Gu, S. Zhao, A. K. Muttyam, and J. M. Hammel, "The Relation Between the Arterial Stress and Restenosis Rate After Coronary Stenting," J. Med. Devices, vol. 4, no. 3, p. 31005, 2010.

[14] S. N. D. Chua, B. J. Mac Donald, and M. S. J. Hashmi, "Finiteelement simulation of stent expansion," J. Mater. Process. Technol., vol. 120, no. 1-3, pp. 335-340, Jan. 2002.

[15] F. Gervaso, C. Capelli, L. Petrini, S. Lattanzio, L. Di Virgilio, and F Migliavacca, "On the effects of different strategies in modelling balloon-expandable stenting by means of finite element method," $J$. Biomech., vol. 41, no. 6, pp. 1206-1212, 2008.

[16] M. De Beule, P. Mortier, S. G. Carlier, B. Verhegghe, R. Van Impe, and P. Verdonck, "Realistic finite element-based stent design: The impact of balloon folding," J. Biomech., vol. 41, no. 2, pp. 383-389, Jan. 2008.

[17] W. Walke, Z. Paszenda, and J. Filipiak, "Experimental and numerical biomechanical analysis of vascular stent," J. Mater. Process. Technol., vol. 164-165, pp. 1263-1268, May 2005.

[18] S. N. David Chua, B. J. MacDonald, and M. S. J. Hashmi, "Finite element simulation of slotted tube (stent) with the presence of plaque and artery by balloon expansion," J. Mater. Process. Technol., vol. 155-156, pp. 1772-1779, Nov. 2004

[19] C. Lally, F. Dolan, and P. J. Prendergast, "Cardiovascular stent design and vessel stresses: a finite element analysis," J. Biomech., vol. 38, no. 8, pp. 1574-1581, Aug. 2005.

[20] W. Wu, W.-Q. Wang, D.-Z. Yang, and M. Qi, "Stent expansion in curved vessel and their interactions: A finite element analysis," $J$. Biomech., vol. 40, no. 11, pp. 2580-2585, Jan. 2007.

[21] N. Li and Y. Gu, "Parametric design analysis and shape optimization of coronary arteries stent structure," Proc. 6th World Congr. Struct. Multidiscip. Optim. Rio Jan., vol. 30, 2005.

[22] A. Schiavone and L. G. Zhao, "A study of balloon type, system constraint and artery constitutive model used in finite element simulation of stent deployment," Mech. Adv. Mater. Mod. Process., vol. 1, no. 1, Dec. 2015. 